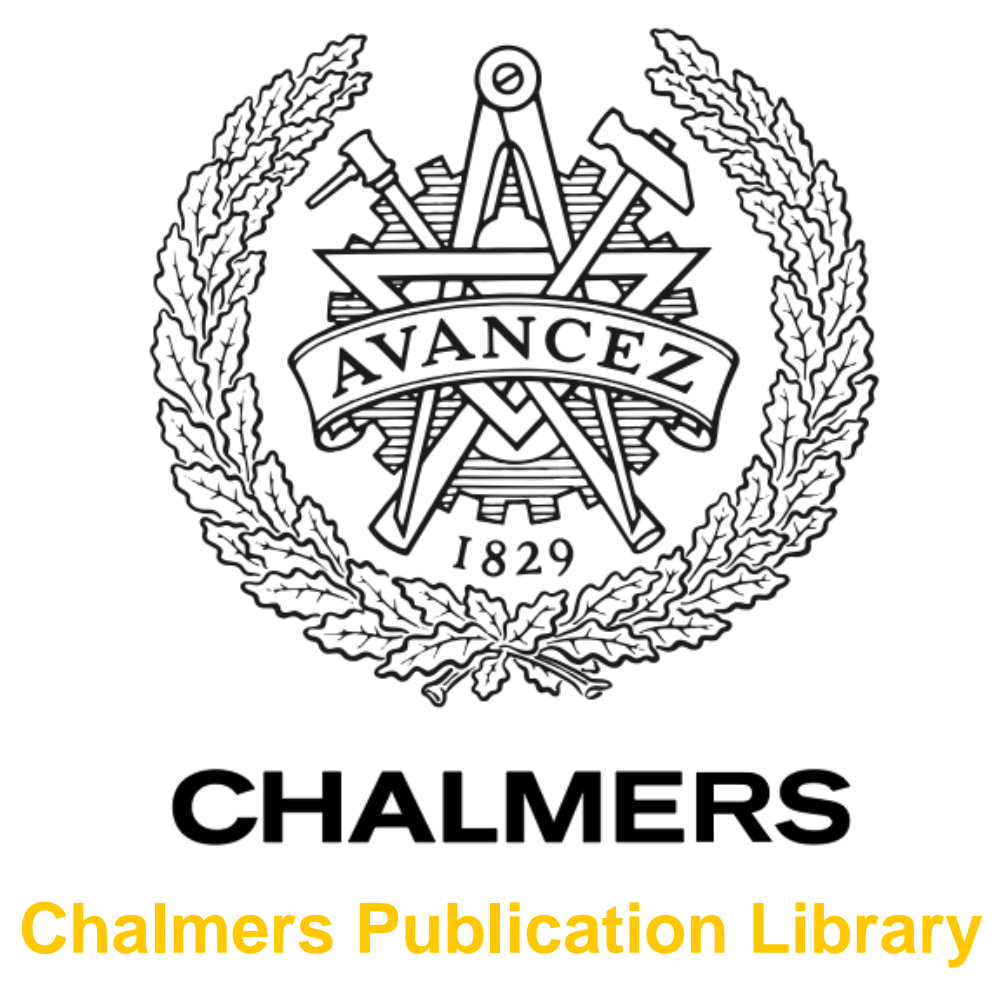

\title{
Challenges for cooperative ITS: Improving road safety through the integration of wireless communications, control, and positioning
}

This document has been downloaded from Chalmers Publication Library (CPL). It is the author's version of a work that was accepted for publication in:

2015 International Conference on Computing, Networking and Communications, ICNC 2015

Citation for the published paper:

Wymeersch, H. ; Rodrigues de Campos, G. ; Falcone, P. et al. (2015) "Challenges for cooperative ITS: Improving road safety through the integration of wireless communications, control, and positioning". 2015 International Conference on Computing, Networking and Communications, ICNC 2015 pp. 573-578.

http://dx.doi.org/10.1109/ICCNC.2015.7069408

Downloaded from: http://publications.lib.chalmers.se/publication/217017

Notice: Changes introduced as a result of publishing processes such as copy-editing and formatting may not be reflected in this document. For a definitive version of this work, please refer to the published source. Please note that access to the published version might require a subscription. 


\title{
Challenges for Cooperative ITS: Improving Road Safety Through the Integration of Wireless Communications, Control, and Positioning
}

\author{
Henk Wymeersch, Gabriel Rodrigues de Campos, Paolo Falcone, Lennart Svensson, Erik G. Ström \\ Department of Signals and Systems, Chalmers University of Technology, 41296 Gothenburg, Sweden \\ email: \{henkw,gabriel.campos,paolo.falcone,lennart.svensson,erik.strom\}@chalmers.se
}

\begin{abstract}
For intelligent transportation systems (ITS) to achieve situational awareness beyond their sensing horizon and to harness coordination capabilities, some form of cooperation will be required. Such cooperation is enabled by vehicle-to-vehicle and vehicle-to-infrastructure wireless communication. The integration between the communication, signal processing, and control sub-systems is non-trivial and requires a co-design, which in turn requires collaboration between these three disciplines. This paper presents a possible evolution of these three disciplines within the context of ITS, as well as several challenges and opportunities.
\end{abstract}

\section{INTRODUCTION}

Intelligent transportation systems (ITS) are widely accepted to enable safer, smarter, and greener transportation systems. In particular, autonomous vehicles are emerging to represent the future of transportation systems and are now a priority for government transportation agencies and vehicle manufacturers, as exemplified through competitions such as the DARPA Urban Challenge [1] and the Grand Cooperative Driving Challenge [2]. Furthermore, several manufacturers have publicly announced their efforts towards fully autonomous vehicles. The main motivations for ITS are as follows. First of all, traffic accidents are responsible for a large number of causalities worldwide. According to several sources, including the World Health Organization, the yearly total number of road deaths is estimated at 1.2 million, with a further 50 million injured every year, though per capita numbers vary significantly from country to country [3]. ITS can aid in reducing these numbers and improving safety of vulnerable road users such as pedestrians, cyclists, and motorcyclists. Secondly, due to the worldwide growing fleet of vehicles and the development of macro-cities, there is a great interest in strategies that can significantly alleviate traffic congestion in urban areas. This last point is important, since congestion generally causes heavy pollution, economic losses, and results in significant waste of energy. ITS can address these challenges through more efficient utilization of the road infrastructure. Finally, ITS will enable new, disruptive transportation solutions, through highly efficient car-sharing, intelligent parking, and providing improved mobility for elderly and children.

The history of ITS can be traced back to the 1970's, following the development of the antilock braking system (ABS) or the more recent electronic stability control [4], both of which are now basic features in passenger cars.
In turn, these gave rise to several methods for improving vehicle handling and stability, such as active front steering and differential braking. More complex maneuvers such as automated takeovers [5] as well as trajectory generation and controller design for longitudinal and lateral movement for vehicle following and collision avoidance [6]-[8] stem from early developments in adaptive cruise control. Finally, strategic decision making procedures for automated highway driving, considering lane change and overtake maneuvers have been treated [9], as well as threat assessment algorithms for lane departure [10].

Though the experimental successes of autonomous vehicles may give the impression that the main problems in ITS have been solved, several challenges remain [11]. First of all, current autonomous vehicles, such as the Google Self-Driving Car, rely on an expensive suite of sensors and a powerful on-board processor. In order to be commercially viable, these must be replaced by fewer, cheaper and thus more unreliable sensors, combined with small, power-efficient processing units performing data fusion [12]. Secondly, the situational awareness of each autonomous vehicle is limited to the range of its sensors and map-based context information, thus reducing the possibilities to optimize its motion to the surrounding traffic situation as well as the efficiency and safety of traffic flow, or to reduce traffic congestion or fuel consumption. To address these challenges, the idea of cooperative ITS has been put forth [13], where vehicles communicate wirelessly with nearby road users to share state and control information. The range of applications of cooperative ITS is large, including cooperative platoon control [14], cooperative ramp metering [15], conflict resolution at traffic intersections [16]-[19], or vehicle re-routing [20].

The heterogeneity of applications imposes a variety of demands not only on the control algorithms, but also on the signal processing/sensing units, and the wireless communication system. However, most of the aforementioned works have been approached within a given discipline, ignoring or simplifying the limitations set by the other disciplines. From a control point of view, for instance, decisions are often made under the assumption of perfect information exchange, precise measurements, or oversimplified communication models. The study of communication systems generally ignores the 
requirements of the control algorithm, focusing exclusively on minimizing packet losses and delays. Finally, sensing information is typically ignorant of how it will be used, and what the current accuracy or freshness requirements are.

In this paper, we will elaborate on several expected challenges in cooperative ITS, both within the individual disciplines as well as across them. These challenges can serve as inspiration for educators, researchers, and practicing engineers, to stimulate closer collaboration across disciplinary boundaries.

\section{A Representative Problem: Intersection CROSSING}

A particularly challenging problem is traffic control near intersections, which are among the most complex and accidentprone elements of modern traffic systems, accounting for $43 \%$ of the total injury-causing accidents and $21 \%$ of the vehiclerelated fatalities in Europe [3]. Consequently, intersections are among the most controlled traffic infrastructures, often regulated simultaneously by right-of-way rules, signs, and traffic lights. The complexity and comprehensive regulation means that traffic intersections often form bottlenecks in the traffic system, where the average speed and traffic throughput drop significantly.

Cooperative ITS have the potential to improve traffic flow and safety near intersections, without relying on inefficient traffic lights or error-prone human control (as depicted in Fig. 1). Instead, vehicles equipped with communication devices, have to coordinate and agree on how to cross the intersection without collisions. Ideally, by exploiting their communication capabilities, the vehicles should be able to coordinate and achieve a quality of service requirement, such as the minimization of the aggregate fuel-consumption (e.g., by slowing down a light vehicle instead of a bus or a heavy truck). However, intersection crossing with cooperative ITS is far from simple. From a control perspective, coordination is a multi-agent control problem that is generally non-convex and often NP-complete. From a communication perspective, the intersection corresponds to a dense, mobile ad-hoc network, with stringent latency requirements. From a signal processing perspective, obtaining state information requires dealing with a mobile, highly complex environment with limited visibility. Together, this makes the intersection scenario important (and arguably representative of cooperative ITS), presenting many of the challenges inherent to other scenarios.

While intersections such as the one presented in Fig. 1, where all vehicles are part of the cooperative ITS, are unlikely to materialize for many years to come, they indicate an evolution from our current state. In particular, we break down this evolution into four phases.

1) Phase 1: This phase represents the present, where there are very few autonomous vehicles and the remaining vehicles have no wireless communication capabilities.

2) Phase 2: In this phase, there are still very few autonomous vehicles, but most vehicles have support for wireless communications systems. These systems allow

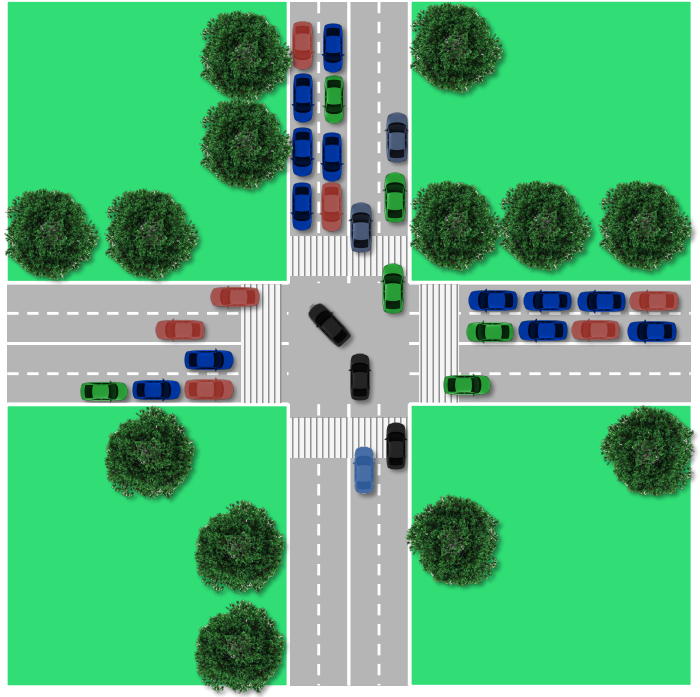

Fig. 1. An example of a complex intersection, where the traffic lights have been removed. Autonomous vehicles must communicate with nearby vehicles to share state and control information in order to safely and efficiently cross the intersection.

sharing of state (e.g., position, velocity, intention) information.

3) Phase 3: In the third phase, a majority of vehicles will be autonomous, with only very few legacy humandriven vehicles remaining. The autonomous vehicles will have the opportunity to share control information, in addition to state information (which can be shared by all vehicles).

4) Phase 4: Finally, all vehicles are autonomous. The entire system now relies exclusively on sharing control and state information, without any human intervention.

\section{Evolution IN CONTROL, COMMUNiCATIONS, AND SigNAL PROCESSING}

In order to design and implement coordination strategies for each of the four phases, a number of challenges need to be addressed. These challenges relate not only to control theory (required to plan, optimize, and coordinate actions of vehicles), but also to wireless communications (to share information about position, velocity, intentions, and control actions), and signal processing (to estimate and predict vehicles' states, the state of the environment, and their relation to underlying maps). Each of the four phases poses specific challenges for each of these three disciplines, as depicted in Fig. 2. In this section, we will present several challenges faced by the three disciplines as ITS transition from Phase 1 to Phase 4.

\section{A. Control Theory}

In Phase 1, an autonomous vehicle has to cross the intersection in the presence of other vehicles relying on onboard sensors only. This leads to a number of problems: the autonomous vehicle has to (i) model/determine the intention of the surrounding vehicles; (ii) predict the motion of these vehicles; (iii) cross the intersection, given the predicted motion of the non-autonomous vehicles. The first and second problems can be solved jointly by modeling a non-autonomous vehicle with 


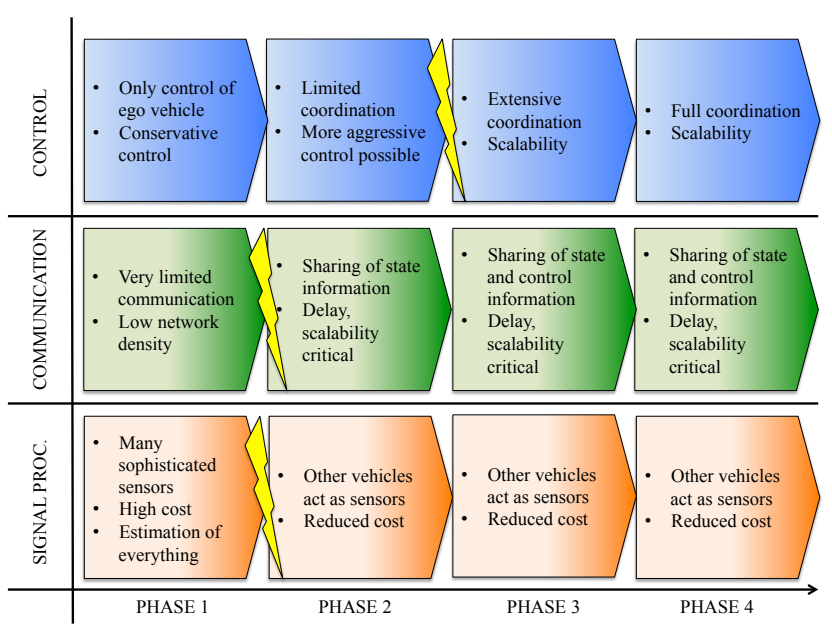

Fig. 2. Illustration of the four-phase evolution in the different disciplines. The lightning bolts represent a significant change within that discipline. In control theory, the transition from Phase 2 to Phase 3 will require scalable algorithms. In wireless communication, the sharing of information among all vehicles occurs in the transition from Phase 1 to Phase 2. In signal processing, the strain on the sensor sub-system is reduced in the same transition, due to the availability of state information of all vehicles.

a simplified dynamic model, based on which a Kalman filter can be designed. Alternatively, the two problems can be solved separately based in an hybrid approach, in which a vehicle is modeled by several continuous dynamical systems and a finite automaton describing the transitions between different modes [21]. In case other autonomous vehicles are present near the intersection, all autonomous vehicles should agree on (i) and (ii) in order to solve (iii). Agreement can be achieved either in a centralized manner (by letting a central traffic control unit collect information via wireless communication and fuse the predictions calculated by each autonomous vehicle), or in a distributed manner (using some form of distributed consensus [22], [23]). Finally, negotiating the intersection can in principle be formulated either in a centralized or distributed setting [16], [24]. In both cases, the resulting control problem has to account for the uncertainty in the predicted motion of the non-autonomous vehicles. Robust control frameworks such as min-max model predictive control [25] are recognized to lead to a conservative solution, and may even lead to infeasible problems. Alternatively, stochastic optimal control frameworks [26], [27] can lead to less conservative coordination.

From Phase 2 onward, the autonomous vehicles can have access to state and intention information of most surrounding vehicles. In that case, the importance of intention detection and motion prediction can be reduced for those vehicles. By agreeing on the maximum deviation of non-autonomous vehicles from the intended paths or a a corresponding distribution over a future finite time horizon, the intersection can be safely crossed. Several intervention-based solutions have been proposed to cope with the coexistence of human-driven and autonomous vehicles. More specifically, [24], [28] present a supervisory controller that overrides the driver's commands if these take the vehicle out of the maximum control invariant set with respect to a collision inside the intersection. It can be shown that this control problem is NP-hard [24], but that a polynomial time approximation scheme exists. This intervention-based approach is elaborated in [17], [29], where experimental results are provided. Note that due to safety requirements, control algorithms must still function in case information exchange is not possible. This could be achieved, for instance, through more advanced signal processing algorithms or more robust control units.

Phases 3 and 4 are characterized by a growth in the size of the control problem. Hence, control strategies should scale gracefully with the number of vehicles. Interestingly, a great deal of the literature has focused on Phase 4, and several studies have considered cooperative strategies for intersection scenarios, though generally abstracting from the wireless communication and signal processing aspects. Decentralized approaches based on predictive control and reachability analysis [30], [31] and on navigation function controllers [32] have been considered. A somewhat different approach is taken in [33], where the dynamics are abstracted away and a number of protocols for distributed decision making are presented and compared. A partly centralized, provably safe strategy is presented in [16] based on centralized scheduling and subsequent time-slot assignment for individual vehicles. Related ideas are explored in detail in [34].

\section{B. Wireless Communications}

In the above works, limitations set by wireless communication between the involved vehicles and/or the central infrastructure are not directly addressed, meaning that the problems inherent to wireless communication (e.g., packet losses and delays) are largely ignored. On the other hand, research on vehicular communication has mainly focused on measurement campaigns and simulations for vehicular channel characterization without considering detailed requirements of specific applications [35], [36]. It is clear that different applications within the vehicular domain have different requirements on the communication links and that safety-related applications considered in this paper are among the ones imposing the most stringent demands, requiring extremely low latencies, high delivery ratios for full situational awareness, and relatively long communication ranges to increase the time to react in critical situations.

Challenges for wireless communication stem from the need to provide low latency, high reliability, efficient use of spectrum, and high availability. These challenges are easily addressed in Phase 1, due to the low number of vehicles equipped with communication capabilities. In contrast, in Phases 2, 3 , and 4 , the dense and rapidly varying network topologies (due to mobility) put a strain on the wireless communication capabilities, so that careful trade-offs need to be made. The issue of low latency and high availability motivates the use of an ad-hoc network topology without central controllers (e.g., base stations or access points), as a central controller implies a single point of failure and increased latency due to control traffic and/or relaying of messages through the central controller (note that control traffic refers to digital data used to assign resources and coordinate the wireless transmission; it 
should not be confused with control algorithms from Section III-A). The local broadcast nature of the communication and fast changing network topology makes it hard or expensive to provide reliability. The standard approach based on retransmission protocols is not attractive due to the potentially excessive feedback data traffic and uncertainties in who the receivers actually are.

Current standards for vehicle-to-vehicle (V2V) and vehicleto-infrastructure (V2I) communication are based on the physical (PHY) and medium access control (MAC) layers specified by IEEE $802.11 \mathrm{p} \mathrm{[37],} \mathrm{[38].} \mathrm{It} \mathrm{is} \mathrm{noteworthy} \mathrm{that} 802.11 \mathrm{p}$ is an amendment to the 802.11 standard used in $\mathrm{WiFi}$, and thus the PHY and MAC layers are optimized for low-mobility, indoor propagation environments with best-effort data traffic. Given the requirements for cooperative ITS (high-mobility, outdoor propagation with periodic or event-driven data traffic with stringent latency and reliability requirements), it is therefore no surprise that $802.11 \mathrm{p}$ technology can be improved upon with a clean-slate approach. The main advantage of $802.11 \mathrm{p}$ is that it allows for a truly ad-hoc network topology, required for control and signal processing algorithms that operate in a fully distributed manner. Hence, the time-consuming operation of attaching nodes to a centralized unit is avoided, at the cost of increased security overhead. However, for current spectrum allocation, predicted data traffic demands, and due to the 802.11 MAC relatively poor scaling behavior, channel congestion is a very clear possibility and efforts are made to define efficient distributed congestion control protocols [39].

As an alternative or complement to $802.11 \mathrm{p}, 5 \mathrm{G}$ communication systems are considered for low-latency device-todevice (D2D) communication for V2V and V2I [40]. There are several challenges associated with this approach, chiefly how to limit interference between "regular" non-D2D cellular users and the D2D users. For the D2D links to be useful for cooperative ITS, the associated control traffic from the base stations must be designed such that D2D nodes can communicate with low latency and also under conditions when there is limited or no network connectivity.

\section{Signal Processing}

Signal processing mainly deals with the design and implementation of the sensor fusion system, required to provide situational awareness to the control application. More specifically, the sensor fusion system provides information about the environment around the own vehicle, the positions of surrounding objects and vehicles, the shape and topology of the road, and to some extent also traffic rules (e.g., who has right of way).

In Phase 1, autonomous vehicles (such as those currently demonstrated by various universities and companies) typically rely on an expensive, highly accurate sensor suite, comprising a combination of vehicle motion sensors (e.g., accelerometers, wheel odometers) [41], a global navigation satellite system (GNSS) [42], and external sensors (e.g., high-end cameras, LIDAR or RADAR) that can observe surrounding landmarks and dynamic objects [43], [44]. In addition, a detailed map of the road and the static landmarks is normally created in advance, in order to enable the fusion system to make use of the observations from the landmarks to obtain high-precision estimates of the vehicle position [45]-[47]. Sensor fusion systems face a number of challenges: they need to provide highly accurate position estimates of the own vehicle, as well as other road users in its vicinity; external sensors are limited in terms of coverage (i.e., they cover a limited distance and angle, especially around intersections where occlusions are common); and they must be able to detect and classify a wide range of road users and objects, including other vehicles. Another considerable challenge is related to traffic rules, part of which can be encoded through static map annotations [48], but another part involves dynamic variations (e.g., road construction, a police officer who temporarily takes over the control of the traffic) that must be accounted for during sensor fusion.

A major change in the sensor system occurs when we transition into Phase 2. The availability of V2V and V2I communication, e.g., "Here-I-Am"-messages (called basic safety messages (BSMs) or cooperative awareness messages (CAMs) in the US and Europe, respectively) along with information regarding traffic lights can reduce the demands on the sensor fusion system, since many actors in the environment can transfer their state to the own vehicle, rather than having the own vehicle estimate these states [2], [14]. Information about landmarks, pedestrians, bicyclists, and animals can also be shared, reducing risks of sensor occlusion. Finally, V2I communication can explicitly inform vehicles about nearby construction sites or the state of the traffic lights. Nevertheless, in order to ensure continuity of service, vehicles may not rely solely on the presence of other vehicles in order to obtain the required state information, and must still be equipped with sensors to independently detect pedestrians, bicyclists, and other unexpected objects, in order to be able to drive autonomously when no other vehicles are present.

Fusion of information among vehicles comes with its own set of technical opportunities and challenges. For instance, it is highly desirable to use the communication system in order to enable vehicles to improve the estimates of their own positions using measurements with respect to other vehicles. One example is the improved robustness and redundancy that can be obtained in intersections by sharing landmark observations among all vehicles. To our knowledge, such systems have not been properly investigated for autonomous vehicles, but related problems have been studied in other fields. In particular, a general method to perform cooperative localization in wireless networks was proposed in [49], relying on inter-vehicle distances and message passing algorithms. Agreement regarding global positions of landmarks and maps can be accomplished in a principled manner using distributed consensus algorithms [23], which have been studied extensively in the control community. Nevertheless, application of the ideas from [23], [49] to a traffic environment may still be very challenging. 


\section{TOWARd AN INTEgRated ARChitecture}

The above section highlights the fact that intelligent collision avoidance techniques rely on a complex, structured and hierarchical interaction between the different disciplines. Indeed, it is clear that the signal processing/sensor fusion system is a service to control system, providing the control system with the necessary information regarding vehicles and obstacles in order formulate a suitable control problem and to coordinate crossing of the intersection. In turn, the signal processing system relies on services from the communication system, as sensor fusion relies on state information communicated by nearby vehicles, and possibly for message passing or consensus algorithms to improve state estimates. Finally, the wireless communication system is a service to the control system, as agreement regarding the control actions relies on data exchange between all vehicles. These relationships are visualized in Fig. 3, highlighting also the fact that each of the disciplines relies on real-time computing to execute all of the algorithms. Below, we identify several design considerations that relate to the interfaces between the different disciplines.

\section{A. Control and Signal Processing}

Control strategies must be designed in such a way that state uncertainties are accounted for explicitly. Overly optimistic assumptions in the control system will lead to unsafe operation. Overly pessimistic assumptions will lead to poor performance due to unduly conservative control strategies. In either case, improper use of sensor fusion information will cancel out many of the possible gains of cooperative ITS. This requires new optimal control formulations with explicit incorporation of statistical sensing models. By accounting for the underlying sensing and prediction methods, advantages could arise from using uncertainty information, not only on current estimates but also on future ones, in the design of robust control algorithms. In addition, the control system impose requirements that the sensor fusion output should satisfy. Such requirements provide useful guidelines, e.g., when selecting sensors.

\section{B. Control and Communications}

Modern control algorithms rely on an optimization horizon, which is limited by the sensing range. Through wireless communication the sensing range can be greatly increased, thus allowing a longer optimization horizon. However, when control relies on communication, either between vehicles or through a centralized unit, control strategies should be designed to accommodate packet losses and communication delays. In particular, algorithms should degrade gracefully with packet losses, instead of failing all together, and should be built on realistic assumptions of the communication system. In addition, coherent fall-back plans should be developed in case the communication cannot support the demands of the control strategy. This requires new optimal control formulations with explicit incorporation of wireless communication models. In turn, the communication protocols must be developed with performance guarantees in terms of latency and availability, possibly adapting with increased network density. For instance, distributed optimization algorithms should be evaluated under realistic wireless channel conditions and protocol assumptions.

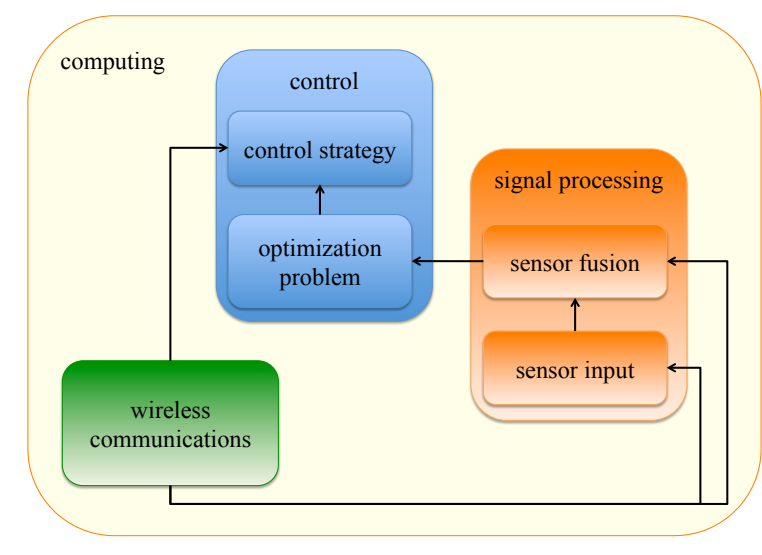

Fig. 3. Simplified interaction between the different disciplines, where arrows represent "provides a service for". The aspect of real-time computation was not considered in this paper, but is mandatory to realize the control algorithms, sensor fusion, and wireless communications.

\section{Signal Processing and Communications}

While wireless communication can significantly increase the sensing range of individual vehicles, due to the unreliable nature of the wireless link, sensor fusion must account for delays, outages, and out-of-order measurements. Similar to distributed control strategies, distributed sensor fusion algorithms must be designed assuming realistic performance of the communication system, and allowing graceful degradation with packet losses.

\section{CONCLUSiOnS AND OUTLOOK}

As intelligent transportation systems (ITS) transition from solitary autonomous vehicles to full-fledged cooperative systems, significant societal benefits will emerge. Progress in this area relies on interaction between the areas of control theory, wireless communication, and signal processing. This paper has given an overview of how these disciplines are expected to evolve within the context of cooperative ITS, and how they inter-relate. These inter-dependencies call for more integration between these disciplines, both at the research level and in engineering education.

\section{ACKNOWLEDGMENTS}

This research was supported, in part, by the European Research Council under Grant No. 258418 (COOPNET), Chalmers' Area of Advance in Transportation, SAFER-Vehicle and Traffic Safety Center and the Department of Signals and Systems (S2). The authors would like to thank Erik Steinmetz and Robert Hult for their careful proofreading of the paper.

\section{REFERENCES}

[1] "DARPA Urban Challenge," http://archive.darpa.mil/grandchallenge/, 2007.

[2] “Grand Cooperative Driving Challenge," http://www.gcdc.net/, 2010.

[3] M. Simon, T. Hermitte, and Y. Page, "Intersection road accident causation: A European view," $21^{\text {st }}$ International Technical Conference on the Enhanced Safety of Vehicles, pp. 1-10, 2009.

[4] A. Eidehall, Tracking and threat assessment for automotive collision avoidance. $\mathrm{PhD}$ thesis, Linköping University, 2007.

[5] A. Vahidi and A. Eskandarian, "Research advances in intelligent collision avoidance and adaptive cruise control," IEEE Transactions on Intelligent Transportation Systems, vol. 4, no. 3, pp. 143-153, 2003. 
[6] C. Hatipoglu, U. Ozguner, and K. Redmill, "Automated lane change controller design," IEEE Transactions on Intelligent Transportation Systems, vol. 4, pp. 13-22, March 2003.

[7] S. J. Anderson, S. C. Peters, T. E. Pilutti, and K. Iagnemma, "An optimalcontrol-based framework for trajectory planning, threat assessment, and semi-autonomous control of passenger vehicles in hazard avoidance scenaria," International Journal of Vehicle Autonomous Systems, vol. 8, no. $2 / 3 / 4$, pp. $190-216,2010$.

[8] A. Khodayari, A. Ghaffari, S. Ameli, and J. Flahatgar, "A historical review on lateral and longitudinal control of autonomous vehicle motions," in $2^{\text {nd }}$ International Conference on Mechanical and Electrical Technology, pp. 421-429, 2010.

[9] J. Nilsson, M. Ali, P. Falcone, and J. Sjöberg, "Predictive manoeuvre generation for automated driving," in IEEE Conference on Inteligent Transportation Systems, 2013.

[10] M. Ali, P. Falcone, C. Olsson, and J. Sjöberg, "Predictive prevention of loss of vehicle control for roadway departure avoidance," IEEE Transactions on Intelligent Transportation Systems, vol. 14, no. 1, pp. 56-68, 2013.

[11] I.-Y. Hsu, M. Wodczak, R. White, T. Zhang, and T. Hsing, "Challenges, approaches, and solutions in intelligent transportation systems," in Second International Conference on Ubiquitous and Future Networks (ICUFN), pp. 366-371, 2010.

[12] N.-E. E. Faouzi, H. Leung, and A. Kurian, "Data fusion in intelligent transportation systems: Progress and challenges - a survey," Information Fusion, vol. 12, no. 1, pp. $4-10,2011$. Special Issue on Intelligent Transportation Systems.

[13] S. Behere, M. Törngren, and D.-J. Chen, "A reference architecture for cooperative driving," Journal of Systems Architecture, vol. 59, no. 10, Part C, pp. 1095 - 1112, 2013.

[14] R. Kianfar, B. Augusto, A. Ebadighajari, U. Hakeem, J. Nilsson, A. Raza, R. S. Tabar, N. Irukulapati, C. Englund, P. Falcone, et al., "Design and experimental validation of a cooperative driving system in the grand cooperative driving challenge," IEEE Transactions on Intelligent Transportation Systems, vol. 13, no. 3, pp. 994-1007, 2012.

[15] R. Scarinci, B. Heydecker, and A. Hegyi, "Analysis of traffic performance of a ramp metering strategy using cooperative vehicles," in IEEE Conference on Intelligent Transportation Systems, pp. 324-329, 2013.

[16] H. Kowshik, D. Caveney, and P. Kumar, "Provable systemwide safety in intelligent intersections," IEEE Transactions on Vehicular Technology, vol. 60, no. 3, pp. 804-818, 2011.

[17] M. Hafner, D. Cunningham, L. Caminiti, and D. D. Vecchio, "Cooperative collision avoidance at intersections: Algorithms and experiments," IEEE Transactions on Intelligent Transportation Systems, vol. 14, no. 3, pp. 1162-1175, 2013.

[18] K.-D. Kim, "Collision free autonomous ground traffic: A model predictive control approach," in $4^{\text {th }}$ ACM/IEEE International Conference on Cyber-Physical Systems, 2013.

[19] L. Makarem and D. Gillet, "Model predictive coordination of autonomous vehicles crossing intersections," in IEEE Conference on Inteligent Transportation Systems, 2013.

[20] S. Boskovich and M. Barth, "Vehicular network rerouting autonomy with a V2V, I2V, and V2I communication matrix classification," in Intelligent Transportation Systems - (ITSC), 2013 16th International IEEE Conference on, pp. 172-177, 2013.

[21] R. Verma and D. D. Vecchio, "Safety control of hidden mode hybrid systems," IEEE Transactions on Automatic Control, vol. 57, no. 1, pp. 62-77, 2012.

[22] S. Kar and J. Moura, "Distributed consensus algorithms in sensor networks with imperfect communication: Link failures and channel noise," IEEE Transactions on Signal Processing, vol. 57, no. 1, pp. 355$369,2009$.

[23] R. Olfati-Saber, J. A. Fax, and R. M. Murray, "Consensus and cooperation in networked multi-agent systems," Proceedings of the IEEE, vol. 95, no. 1, pp. 215-233, 2007.

[24] A. Colombo and D. Del Vecchio, "Efficient algorithms for collision avoidance at intersections," in $15^{\text {th }}$ ACM International Conference on Hybrid Systems: Computation and Control, 2012.

[25] P. O. M. Scokaert and D. Mayne, "Min-max feedback model predictive control for constrained linear systems," IEEE Transactions on Automatic Control, vol. 43, no. 8, pp. 1136-1142, 1998.

[26] X.-H. Yu and W. Recker, "Stochastic adaptive control model for traffic signal systems," Transportation Research Part C, vol. 14, no. 4, pp. $263-$ 282,2006
[27] A. Bemporad and S. Di Cairano, "Optimal control of discrete hybrid stochastic automata," in Hybrid Systems: Computation and Control, vol. 3414 of Lecture Notes in Computer Science, pp. 151-167, Springer Berlin Heidelberg, 2005.

[28] A. Colombo and D. D. Vecchio, "Enforcing safety of cyberphysical systems using flatness and abstraction," in Work-in-progress session of ICCPS, 2011.

[29] M. R. Hafner, D. Cunningham, L. Caminiti, and D. D. Vecchio, "Automated vehicle-to-vehicle collision avoidance at intersections," in ITS World Congress, 2011.

[30] G. R. Campos, P. Falcone, and J. Sjöberg, "Autonomous cooperative driving: a velocity-based negotiation approach for intersection crossing," in IEEE Conference on Inteligent Transportation Systems, 2013.

[31] G. R. Campos, P. Falcone, H. Wymeersch, J. Sjöberg, and R. Hult, "A receding horizon control strategy for cooperative conflict resolution at traffic intersections," 2014. Submitted to the IEEE Conference on Decision and Control.

[32] L. Makarem and D. Gillet, "Fluent coordination of autonomous vehicles at intersections," IEEE International Conference on Systems, Man, and Cybernetics (SMC), pp. 2557-2562, 2012.

[33] S. R. Azimi, G. Bhatia, and R. R. Rajkumar, "Reliable intersection protocols using vehicular networks," ACM/IEEE 4th International Conference on Cyber-Physical Systems (ICCPS), 2013.

[34] K. Dresner, "A Multiagent Approach to Autonomous Intersection Management," Journal of Artificial Intelligence Research, vol. 31, pp. 591656, 2008

[35] C. F. Mecklenbräuker, A. F. Molisch, J. Karedal, F. Tufvesson, A. Paier, L. Bernado, T. Zemen, O. Klemp, and N. Czink, "Vehicular Channel Characterization and Its Implications for Wireless System Design and Performance," Proceedings of the IEEE, vol. 99, pp. 1189-1212, July 2011.

[36] K. Sjöberg, Medium Access Control for Vehicular Ad Hoc Networks. $\mathrm{PhD}$ thesis, Chalmers University of Technology, 2013.

[37] J. B. Kenney, "Dedicated short-range communications (DSRC) standards in the United States," Proceedings of IEEE, vol. 99, pp. 1162-1182, July 2011.

[38] E. G. Ström, "On medium access and physical layer standards for cooperative intelligent transport systems in Europe," Proc. IEEE, vol. 99, pp. 1183-1188, July 2011. Invited paper.

[39] J. B. Kenney, G. Bansal, and C. E. Rohrs, "LIMERIC: a linear message rate control algorithm for vehicular DSRC systems," in Proc. Eighth International Workshop on Vehicular Ad Hoc Networks, Sept. 2011.

[40] "Scenarios, requirements and KPIs for 5G mobile and wireless system." ICT-317669-METIS/D1.1, METIS deliverable D1.1, Apr. 2013.

[41] I. Skog and P. Händel, "In-car positioning and navigation technologies - a survey," IEEE Transactions on Intelligent Transportation Systems, vol. 10, no. 1, pp. 4-21, 2009.

[42] B. Hofmann-Wellenhof, H. Lichtenegger, and E. Wasle, GNSS - global navigation satellite systems: GPS, GLONASS, Galileo, and more. Springer, 2007.

[43] J. Ziegler, P. Bender, M. Schreiber, H. Lategahn, T. Strauss, C. Stiller, T. Dang, U. Franke, N. Appenrodt, C. Keller, et al., "Making Bertha drive - an autonomous journey on a historic route," IEEE Intelligent Transportation Systems Magazine, vol. 6, no. 2, pp. 8-20, 2014.

[44] A. Vu, J. Farrell, and M. Barth, "Centimeter-accuracy smoothed vehicle trajectory estimation," IEEE Intelligent Transportation Systems Magazine, vol. 5, no. 4, pp. 121-135, 2013.

[45] G. Grisetti, R. Kummerle, C. Stachniss, and W. Burgard, "A tutorial on graph-based SLAM," IEEE Intelligent Transportation Systems Magazine, vol. 2, no. 4, pp. 31-43, 2010.

[46] S. Thrun, M. Montemerlo, H. Dahlkamp, D. Stavens, A. Aron, J. Diebel, P. Fong, J. Gale, M. Halpenny, G. Hoffmann, et al., "Stanley: The robot that won the DARPA grand challenge," Journal of field Robotics, vol. 23, no. 9, pp. 661-692, 2006.

[47] J. Levinson and S. Thrun, "Robust vehicle localization in urban environments using probabilistic maps," in 2010 IEEE International Conference on Robotics and Automation (ICRA), pp. 4372-4378, 2010.

[48] F. Ramm, J. Topf, and S. Chilton, "Openstreetmap." UIT Cambridge. [Online], 2007. Available: http://openstreetmap.info/content/ OpenStreetMap-Kapitel5-Mapping-Praxis.pdf.

[49] H. Wymeersch, J. Lien, and M. Z. Win, "Cooperative localization in wireless networks," Proceedings of the IEEE, vol. 97, Feb. 2009. 